\title{
Correction to: From stand-up to start-up: exploring entrepreneurship competences and STEM women's intention
}

\author{
Cristina Armuña ${ }^{1}$ (D) Sergio Ramos ${ }^{2}$ - Jesús Juan $^{3} \cdot$ Claudio Feijóo $^{4}$. \\ Alberto Arenal ${ }^{1}$ \\ Published online: 1 July 2020 \\ (C) Springer Science+Business Media, LLC, part of Springer Nature 2020
}

Correction to: International Entrepreneurship and Management Journal (2020) 16: 69-92

https://doi.org/10.1007/s11365-019-00627-z

The official affiliation of the corresponding author is Universidad Politécnica de Madrid (UPM).

Publisher's note Springer Nature remains neutral with regard to jurisdictional claims in published maps and institutional affiliations.

The online version of the original article can be found at https://doi.org/10.1007/s11365-019-00627-z

\author{
Cristina Armuña \\ cristinaag@alumnos.upm.es \\ Sergio Ramos \\ sramos@cee.uned.es \\ Jesús Juan \\ jesus.juan@upm.es \\ Claudio Feijóo \\ claudio.feijoo@upm.es \\ Alberto Arenal \\ aarenal@alumnos.upm.es
}

Extended author information available on the last page of the article 


\section{Affiliations}

Cristina Armuña ${ }^{1} \cdot$ Sergio Ramos ${ }^{2} \cdot$ Jesús Juan $^{3} \cdot$ Claudio Feijóo $^{4} \cdot$ Alberto Arenal ${ }^{1}$

1 Universidad Politécnica de Madrid (UPM), Universidad Nacional de Educación a Distancia (UNED), Madrid, Spain

2 Universidad Nacional de Educación a Distancia (UNED), Universidad Politécnica de Madrid (UPM), Madrid, Spain

3 Universidad Politécnica de Madrid (UPM), Madrid, Spain

4 Universidad Politécnica de Madrid (UPM), Madrid, Spain, Tongji University, Shanghai, China 